ARTICLE

Received 2 Feb 2015 | Accepted 4 Jun 2015 | Published 16 Jul $2015 \quad$ DOI: 10.1038/ncomms8730 OPEN

\title{
A generic concept to overcome bandgap limitations for designing highly efficient multi-junction photovoltaic cells
}

Fei Guo ${ }^{1, \star}$, Ning $\mathrm{Li}^{1, \star}$, Frank W. Fecher ${ }^{2}$, Nicola Gasparini ${ }^{1}$, Cesar Omar Ramirez Quiroz ${ }^{1}$, Carina Bronnbauer ${ }^{1,3}$, Yi Hou ${ }^{1,3}$, Vuk V. Radmilović ${ }^{4,5}$, Velimir R. Radmilović ${ }^{4,6}$, Erdmann Spiecker ${ }^{4}$, Karen Forberich ${ }^{1}$

\& Christoph J. Brabec $1,2,3$

The multi-junction concept is the most relevant approach to overcome the Shockley-Queisser limit for single-junction photovoltaic cells. The record efficiencies of several types of solar technologies are held by series-connected tandem configurations. However, the stringent current-matching criterion presents primarily a material challenge and permanently requires developing and processing novel semiconductors with desired bandgaps and thicknesses. Here we report a generic concept to alleviate this limitation. By integrating series- and parallel-interconnections into a triple-junction configuration, we find significantly relaxed material selection and current-matching constraints. To illustrate the versatile applicability of the proposed triple-junction concept, organic and organic-inorganic hybrid triple-junction solar cells are constructed by printing methods. High fill factors up to $68 \%$ without resistive losses are achieved for both organic and hybrid triple-junction devices. Series/parallel triple-junction cells with organic, as well as perovskite-based subcells may become a key technology to further advance the efficiency roadmap of the existing photovoltaic technologies.

\footnotetext{
${ }^{1}$ Institute of Materials for Electronics and Energy Technology (i-MEET), Friedrich-Alexander University Erlangen-Nürnberg, Martensstrasse 7, 91058 Erlangen, Germany. ${ }^{2}$ Bavarian Center for Applied Energy Research (ZAE Bayern), Haberstrasse 2a, 91058 Erlangen, Germany. ${ }^{3}$ Erlangen Graduate School in Advanced Optical Technologies (SAOT), Friedrich-Alexander-University Erlangen-Nürnberg, Paul-Gordan-Str. 6, 91052 Erlangen, Germany. ${ }^{4}$ Center for Nanoanalysis and Electron Microscopy (CENEM), Friedrich-Alexander University Erlangen-Nürnberg, Cauerstrasse 6, 91058 Erlangen, Germany. ${ }^{5}$ Innovation Center, Faculty of Technology and Metallurgy, University of Belgrade, Karnegijeva 4, 11120 Belgrade, Serbia. ${ }^{6}$ Nanotechnology and Functional Materials Center, Faculty of Technology and Metallurgy, University of Belgrade, Karnegijeva 4, 11120 Belgrade, Serbia. * These authors contributed equally to this work. Correspondence and requests for materials should be addressed to C.J.B. (email: christoph.brabec@fau.de).
} 
T he power conversion efficiency (PCE) of a single-junction photovoltaic cell is fundamentally constrained by the Shockley-Queisser limit ${ }^{1}$. The record efficiencies of few solar technologies, such as single-crystal silicon, $\mathrm{CuInGaSe}_{2}$, CdTe and GaAs solar cells are constantly shrinking the gap to their fundamental efficiency limits ${ }^{2}$. To push the performances of these solar technologies beyond the Shockley-Queisser limit, several approaches have been proposed, for instance, up-conversion ${ }^{3}$, multi-junction configuration ${ }^{4-6}$, multiple exciton generation ${ }^{7,8}$ and concentrator cells, and so on. Among them, the multi-junction concept is one of the most promising candidates that allows to simultaneously address the two dominant loss mechanisms ${ }^{4}$, namely, sub-bandgap transmission and thermalization losses, which account for $>55 \%$ of the total energy of the solar radiation ${ }^{9}$.

The conventional series-connected multi-junction cells are most successful in permanently enhancing the record efficiencies of the respective solar technologies ${ }^{2}$. However, one distinct drawback of the series-connected configuration is the stringent current-matching criterion, which requires careful bandgap engineering in combination with an excellent control of the thicknesses of the respective subcells. Therefore, many highperformance semiconductors with high external quantum efficiency (EQE) in the NIR absorption range exhibit limited applicability for multi-junction operation, as the perfectly matching semiconductor for the front or back subcells is missing. In contrast to the series-connection, a parallelconnection does not require current matching but instead voltage matching. The principle of voltage matching also constrains a semiconductor's applicability with respect to its bandgap, as well as inherently bears potential performance losses with respect to non-ideal open circuit voltages $\left(V_{\mathrm{OC}}\right)$. However, the parallel-connection is more difficult to adapt and optimize for the high-performance semiconductors with non-tunable bandgaps, such as single-crystal silicon or CdTe.
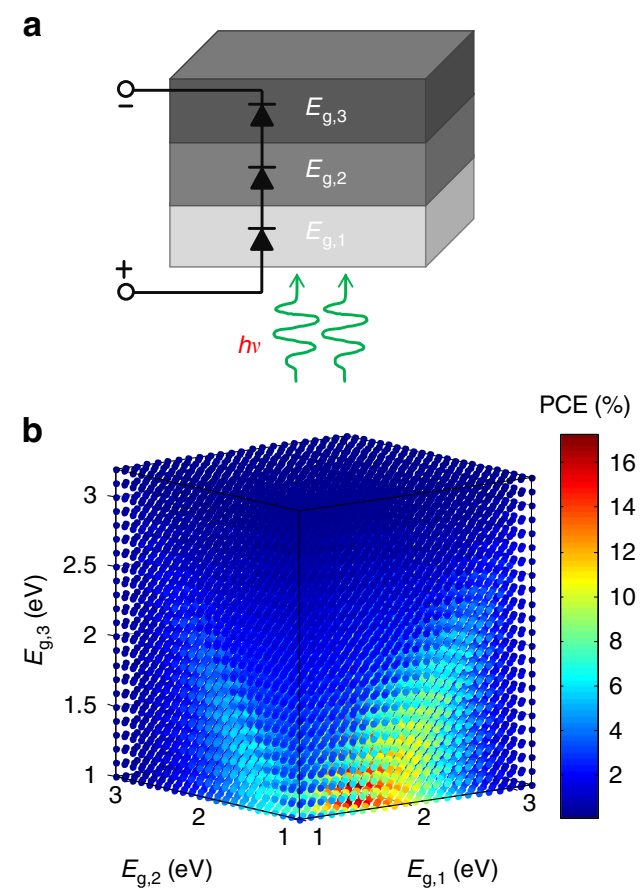

In this manuscript, we present an interconnection approach as a technologically attractive solution to address all these challenges. We propose to deposit a transparent counter electrode and parallel-connect these 'semitransparent' high-efficiency cells with one or more deep NIR sensitizers as back subcells. This is a feasible approach as there are indeed several types of far NIR semiconductors like organic donors ${ }^{10,11}$ and quantum dots ${ }^{12,13}$ with an extended absorption beyond 1,000 $\mathrm{nm}$. The benefit of this series/parallel (SP) multi-junction design is based on the fact that-first, the absorber layer of the front 'semitransparent' hero cell can be made arbitrarily thick (as there is no requirement for current matching), so that this subcell can achieve almost the same efficiency as the opaque single-junction reference. Second, the $V_{\mathrm{OC}}$ of the back cell, which is consisting of a seriesconnection of deep NIR absorbers, can be custom fabricated by stacking an arbitrary sequence of semiconductors with different bandgaps in series. This strategy dramatically reduces the material requirements for voltage matching when parallelconnected to the front subcell. As a consequence, the net photocurrent gain contributed by the deep NIR subcells ultimately adds up to the overall photocurrent of the multijunction photovoltaic cell.

In the following, we start with the demonstration of the integrated SP triple-junction cells for solution-processed organic solar cells. Optical simulations are performed to predict the efficiency potential of different types of triple-junction configurations. Based on rational interface engineering, two fully solution-processed intermediate layers are successively developed, allowing effectively coupling the three cells into a SP interconnected triple-junction configuration. We then extend the concept to the recently emerging perovskite solar cells. A seriesconnected organic tandem solar cell absorbing photons in the NIR range is stacked in a four-terminal configuration behind a semitransparent perovskite cell. Due to the well-matched $V_{\mathrm{OC}}$ between the perovskite cell and the series-connected tandem cell,

C
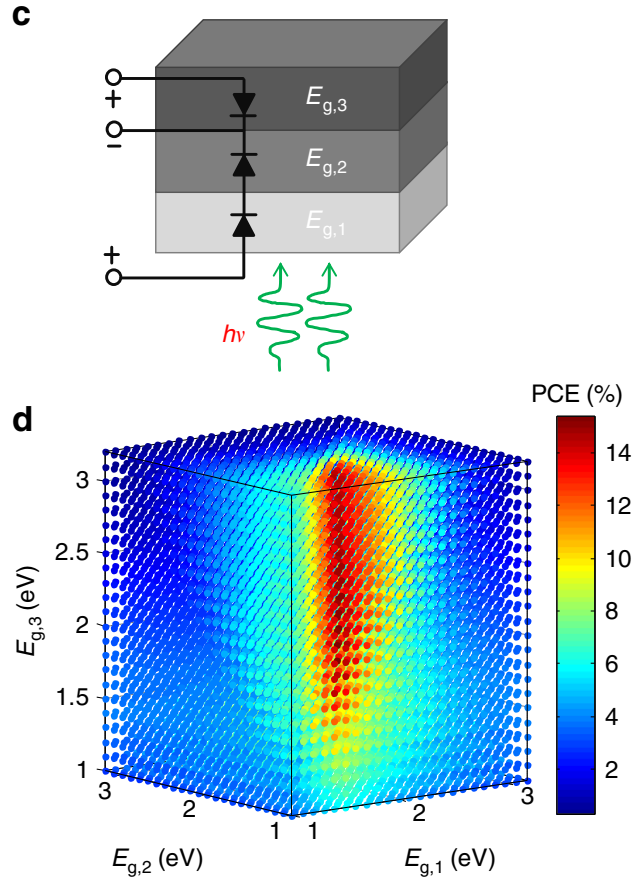

Figure 1 | Comparison of efficiency limit for the two types of triple-junction organic solar cells. (a) Equivalent electronic circuit of the series/series (SS) triple-junction organic solar cells. (b) Three-dimensional efficiency map of the SS triple-junction devices as a function of the absorbers' bandgaps ( $E_{g}$ ) of the three subcells. (c) Equivalent electronic circuit of the series/parallel (SP) triple-junction devices. (d) Three-dimensional efficiency map of the SP triple-junction organic solar cells as a function of the absorbers' bandgaps of the three subcells. 
the photocurrent delivered by the organic tandem cell, up to $2 \mathrm{~mA} \mathrm{~cm}^{-2}$, directly contributes to the performance enhancement of the perovskite cell. Thus, the novel triple-junction concept demonstrated in this work provides an easy but elegant way to manufacture highly efficient photovoltaic cells, not only for conventional but also for the emerging solar technologies.

\section{Results}

Efficiency limits of triple-junction organic solar cells. There are in total four types of device configurations for a triple-junction solar cell, designated as series/series (SS, Fig. 1a), series/parallel (SP, Fig. 1c), parallel/series (PS, Supplementary Fig. 1a) and parallel/parallel (PP, Supplementary Fig. 1b). The SP and PS configurations are distinguished by the stacking sequence of the two interconnections (parallel and series) depending on which interconnection the light passes through first. For organic solar cells, we followed the model proposed by Dennler et al. ${ }^{14,15}$ to calculate the efficiency potential for the four types of triplejunction architectures as a function of the bandgaps of three absorbers. Detailed assumptions and calculation procedure are presented in the Supplementary Note 1. The outcome of the calculations showed that maximum efficiencies of $17.29 \%$, $17.89 \%, 15.41 \%$ and $13.95 \%$ are achievable for SS, PS, SP and PP configurations, respectively. Comparing the four possible interconnections, although the SS and PS configurations demonstrate higher maximum efficiencies, it is apparent that the SP and PP interconnections could offer a wider range of material combinations to reach their highest efficiencies. From a practical point of view, however, the $\mathrm{PP}$ interconnection is too complex to process due to the necessity of introducing two transparent intermediate electrodes. Accordingly, the SP interconnection provides a more feasible approach to reach its theoretical efficiency limit. Hereafter, we shall experimentally show that the SP triple-junction configuration can be fabricated with the intermediate electrode and all the semiconducting layers solution-processed.

Fabrication of bottom DPP-DPP subcell. We began the fabrication of the SP triple-junction devices by designing and processing a semitransparent series-connected double-junction solar cell, as shown in Fig. 2a. We chose a diketopyrrolopyrrolebased low bandgap polymer pDPP5T-2 (abbreviated as DPP) blended with $[6,6]$-phenyl- $\mathrm{C}_{61}$-butyric acid methyl ester $\left(\mathrm{PC}_{60} \mathrm{BM}\right)$ as the photoactive layer of the two front subcells ${ }^{16,17}$, because the main absorption of this heterojunction extends to the near-infrared range with an absorption minimum between
450 and $650 \mathrm{~nm}$ (Supplementary Fig. 2). This absorption characteristic allows the transmitted photons to be absorbed by a wider bandgap top subcell. For series-connected tandem solar cells, the essential component is to construct an efficient intermediate layer serving as charge recombination zone for electrons and holes generated from subcells ${ }^{6,18-25}$. Herein, we chose $\mathrm{ZnO}$ and neutral PEDOT:PSS (N-PEDOT) as the N- and P-type charge extraction materials, respectively, because the work functions of the two materials match well with the energy levels of the donor DPP and acceptor $\mathrm{PC}_{60} \mathrm{BM}^{20,23}$. The transmittance spectrum of $\mathrm{ZnO} / \mathrm{N}-\mathrm{PEDOT}$, the first intermediate layer, is depicted in Fig. 2b. The average transmittance of $94.2 \%$ in the range of $350-850 \mathrm{~nm}$ ensures minimal optical losses from these interface layers. To evaluate the as-designed recombination contacts, series-connected reference tandem cells using DPP:PC ${ }_{60} \mathrm{BM}$ as two identical active layers (denoted as DPP-DPP) were first constructed. As shown in Fig. 2c, the as-prepared opaque tandem device with evaporated $\mathrm{Ca} / \mathrm{Ag}$ top electrode $(15 \mathrm{~nm} / 100 \mathrm{~nm})$ shows a fill factor (FF) of $64.3 \%$ along with a $V_{\mathrm{OC}}$ of $1.1 \mathrm{~V}$ being the sum of two single-junction reference cells (Table 1). These results demonstrated the excellent functionality of the $\mathrm{ZnO} / \mathrm{N}$-PEDOT intermediate layer in the series-connected tandem architecture.

Now, the challenge remains to replace the vacuum-deposited metal electrode with a solution-processed, highly transparent electrode without deteriorating the performance of the established subcells beneath. We chose silver nanowires (AgNWs) as the intermediate electrode for our triple-junction devices because of their high transparency and low sheet resistance as well as the facile solution processability ${ }^{26-30}$. Our recent work demonstrated that a thin layer of $\mathrm{ZnO}$ nanoparticles can effectively conduct electrons to the AgNW electrode and, more importantly, enable the deposition of the AgNW electrode by doctor blading from water-based solution. ${ }^{16,19}$ However, both $\mathrm{ZnO}$ and AgNW layers are obviously not compact enough to protect the underlying subcells from solvent infiltration during the top subcell deposition. We have, therefore, additionally introduced a thin $\mathrm{N}$-PEDOT layer between the $\mathrm{ZnO}$ and AgNWs to realize the second intermediate layer consisting of ' $\mathrm{ZnO} / \mathrm{N}-\mathrm{PEDOT} / \mathrm{AgNW}$ ' (second intermediate layer). Optical transmittance spectra of this intermediate layer and the entire semitransparent tandem DPP-DPP solar cell are shown in Fig. 2b. It is worth mentioning that our second intermediate layer with incorporated AgNWs exhibits an average transmittance of $84.5 \%(400-800 \mathrm{~nm})$, which is a distinct advantage over evaporated thin metal films with low transmittance of $\sim 30-50 \%$ as middle electrode in realizing parallel-connection. ${ }^{31,32}$ Noticeably, the semitransparent tandem a

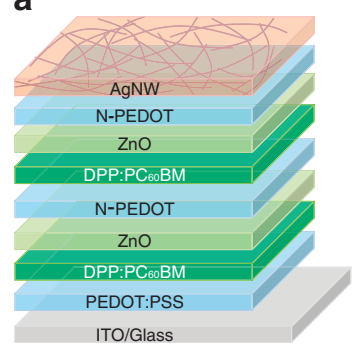

b

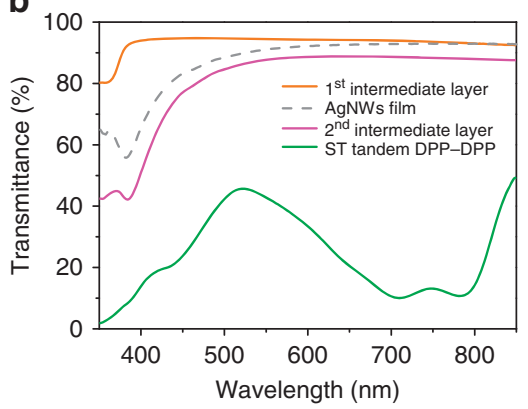

C

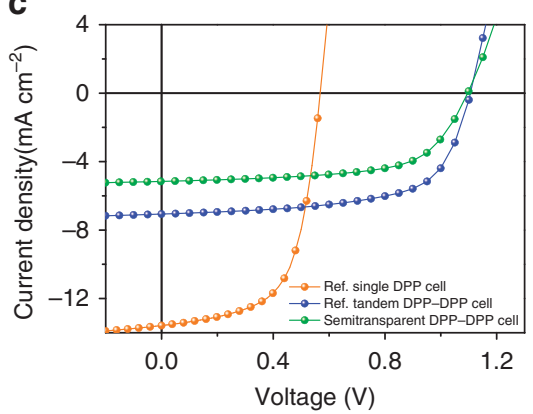

Figure 2 | Semitransparent tandem device structure and performance. (a) Schematic architecture of the semitransparent series-tandem solar cells (DPP-DPP) with AgNWs top electrode. (b) Transmittance spectra of the two intermediate layers used in the SP triple-junction solar cells. (c) Typical J-V curves of the single-junction DPP reference cell, tandem DPP-DPP reference cell and the semitransparent tandem DPP-DPP cell with AgNW top electrode. The device structure of the single and tandem reference cells are: 'Glass/ITO/PEDOT:PSS/DPP:PC 60 BM/Ca/Ag' and 'Glass/ITO/PEDOT:PSS/ DPP:PC ${ }_{60} \mathrm{BM} / \mathrm{ZnO} / \mathrm{N}-\mathrm{PEDOT} / \mathrm{DPP}: \mathrm{PC}_{60} \mathrm{BM} / \mathrm{Ca} / \mathrm{Ag}^{\prime}$. 


\begin{tabular}{|c|c|c|c|c|c|}
\hline Device & $\begin{array}{c}\text { Electrode } \\
\text { bottom/top }\end{array}$ & $\begin{array}{l}V_{\text {oc }} \\
\text { (V) }\end{array}$ & $\begin{array}{l}\mathbf{F F} \\
(\%)\end{array}$ & $\left.\begin{array}{c}J_{S C} \\
(m A ~ c m \\
-2\end{array}\right)$ & $\begin{array}{l}\text { PCE } \\
\text { (\%) }\end{array}$ \\
\hline$D P P: P C_{60} B M$ & $\mathrm{ITO} / \mathrm{Ag}$ & 0.56 & 62.1 & 13.5 & 4.69 \\
\hline Tandem & ITO/Ag & 1.10 & 64.5 & 7.06 & 5.01 \\
\hline DPP-DPP cells & ITO/AgNWs & 1.10 & 63.0 & 5.16 & 3.58 \\
\hline \multirow[t]{2}{*}{ PCDTBT:PC ${ }_{70} B M$} & ITO/Ag & 0.89 & 57.1 & 9.03 & 4.59 \\
\hline & AgNWs/Ag & 0.88 & 55.5 & 8.93 & 4.36 \\
\hline \multirow[t]{2}{*}{ OPV12:PC ${ }_{60} \mathrm{BM}$} & $\mathrm{ITO} / \mathrm{Ag}$ & 0.81 & 67.8 & 9.12 & 5.01 \\
\hline & $\mathrm{AgNW} / \mathrm{Ag}$ & 0.80 & 66.4 & 9.04 & 4.80 \\
\hline
\end{tabular}

DPP-DPP cell shows an average transmittance of $35.6 \%$ in the range of $450-650 \mathrm{~nm}$, which ensures for most wide bandgap materials to be applicable as top subcell to effectively harvest the transmitted photons.

A typical current density versus voltage $(J-V)$ characteristic of the as-prepared semitransparent tandem solar cells (Fig. 2c) exhibits a $V_{\mathrm{OC}}$ of $1.10 \mathrm{~V}$, which is identical to the reference tandem cell, suggesting the effective incorporation of AgNWs as the top electrode. Due to the lack of the back reflective electrode, the semitransparent tandem device shows a relatively low short circuit current $\left(J_{\mathrm{SC}}\right)$ of $5.16 \mathrm{~mA} \mathrm{~cm}^{-2}$. In combination with the still high $\mathrm{FF}$ of $63.0 \%$, these results provide sufficient evidence that the solution-deposited AgNW meshes are highly compatible with the underlying layers without compromising the device performance. In combination with our previous findings that the as-designed intermediate layer was able to resist high boilingpoint solvent rinsing (chlorobenzene and dichlorobenzene) ${ }^{16}$, we expect that the successively established two intermediate layers are capable of coupling the series- and parallel-connected three cells into a monolithically deposited triple-junction stack.

Top subcell fabrication. In our SP triple-junction devices, the top cell is connected in parallel with the bottom series-tandem cell which gives a $V_{\mathrm{OC}}$ of $1.1 \mathrm{~V}$. To match the voltage between the parallel-connected components and thereby maximize the overall efficiency, a top cell with a $V_{\mathrm{OC}}$ value identical or close to the $V_{\mathrm{OC}}$ of the bottom series-tandem cell is desired. Here to demonstrate the general application of our SP triple-junction architecture, we studied two wide bandgap polymers, poly[N-9" ${ }^{\prime \prime}$-hepta-decanyl2,7-carbazole-alt-5,5-(4',7'-di-2-thienyl-2 ${ }^{\prime}, 1^{\prime}, 3^{\prime}$-benzothiadiazole $\left.)\right]$ $\left(\right.$ PCDTBT, $\left.E_{\mathrm{g}}, 1.87 \mathrm{eV}\right)$ and OPV12 $\left(E_{\mathrm{g}}, 1.73 \mathrm{eV}\right)^{33}$, as the top subcells, which give $V_{\mathrm{OC}}$ values of $\sim 0.9 \mathrm{~V}$ and $0.8 \mathrm{~V}$ when mixed with phenyl- $\mathrm{C}_{71}$-butyric acid methyl ester $\left(\mathrm{PC}_{70} \mathrm{BM}\right)$ and $\mathrm{PC}_{60} \mathrm{BM}$, respectively. In addition, as indicated in Supplementary Fig. 2, the absorption profiles of the two active layers are complementary with that of $\mathrm{DPP}: \mathrm{PC}_{60} \mathrm{BM}$, suggesting they are appropriate material combinations for manufacturing multi-junction devices.

To verify the compatibility of the two wide bandgap donors with the AgNW electrode, single-junction reference cells of PCDTBT:PC ${ }_{70} \mathrm{BM}$ and OPV12:PC ${ }_{60} \mathrm{BM}$ were first processed on both indium tin oxide (ITO) and AgNWs-coated glass substrates for comparison (Fig. 3a). Typical $J-V$ characteristics of the asprepared single-junction devices are displayed in Fig. 3b,c and the key photovoltaic parameters are summarized in Table 1. Comparable device performances in terms of $V_{\mathrm{OC}}, J_{\mathrm{SC}}$ and PCE were observed for the two photoactive blends independent of bottom electrode. The slightly lower FFs for the devices fabricated on AgNWs as compared with the ITO counterparts can be ascribed to the higher series resistance $\left(R_{\mathrm{S}}\right)$, probably resulting from the contact resistance between the AgNWs and $\mathrm{ZnO}$. Nevertheless, these results suggest the excellent optoelectronic properties of the AgNWs that are compatible with different polymer donors. Indeed, independent measurement of the AgNW electrode employed in the current study shows an average visible transmittance of $\sim 90 \%$ (Fig. $2 \mathrm{~b}$ ) and a sheet resistance of $\sim 10 \Omega \mathrm{sq}^{-1}$, which is comparable to commonly used ITO electrodes.

Organic triple-junction solar cells. Having successfully constructed the individual bottom semitransparent tandem subcells and top subcell, in combination with the verified robust intermediate layers we now complete the fabrication of the entire SP triple-junction solar cells. Figure $4 \mathrm{a}$ shows the schematic illustration of the SP triple-junction cell design, where the bottom series-connected tandem subcells in a normal structure are electrically connected in parallel with the top inverted subcell. The middle AgNW layer in this triple-junction device serves as a common cathode to collect electrons created by the subcells. It is worth mentioning that we have employed a simple modified doctor blading technique to coat the AgNW electrode ${ }^{16}$, which enables the deposition of the NW film in a stripe and thereby eliminates any subsequent patterning steps. Detailed description of the device fabrication procedure is presented in the Methods section and schematically illustrated in Supplementary Fig. 3.

A cross-sectional transmission electron microscopy (TEM) image of a SP triple-junction solar cell is shown in Fig. 4b. The liftout sample was prepared using a focused ion beam (FIB, FEI Helios NanoLab 660) and imaged subsequently with the TITAN ${ }^{3}$ aberration-corrected TEM. All the individual layers of the solar cell can be clearly distinguished in the scanning TEM (STEM) image without any physical damage. It should be noted that, even though interlayer mixing between the AgNWs and the underlying $\mathrm{N}$-PEDOT layer is observed, it does not negatively affect the device performance since the N-PEDOT in the stack purely acts as a 'solvent protection' layer. On contrary, the fact that the AgNWs partially sink into N-PEDOT can reduce the roughness of the NW networks, which is beneficial for building the upper few layers and further reduces the possibility of shunts in the top subcell. The STEM energy dispersive X-ray spectrometry (EDS) elemental maps (Ag, $\mathrm{Zn}$ and $\mathrm{S}$ ) of the cross-section shown in Fig. 4c confirms a well-organized layer stack.

Simultaneously, optical simulations based on the transfer matrix formalism were carried out to calculate the current generation in the individual subcells ${ }^{34,35}$, which can provide valuable guidance for optimization of our SP triple-junction devices. Taking advantage of the fact that parallel-connection does not require current matching, and therefore balancing the current flow in the bottom series-tandem DPP-DPP cells is of critical significance. In the case of DPP-DPP/PCDTBT triplejunction devices, for the purpose of simplicity we fixed the thickness of the top PCDTBT:PC ${ }_{70} \mathrm{BM}$ to be $80 \mathrm{~nm}$ corresponding to the thickness of optimized single-junction reference cells. Detailed assumption and calculation procedure are presented in the Supplementary Note 2. The outcome of the simulations is shown in Fig. 5a, illustrating the interplay of the photocurrent generation in the three subcells. It is obvious that to maximize the use of incident photons, the thicknesses of the two DPP:PC ${ }_{60} \mathrm{BM}$ active layers should follow the red dashed line where the photocurrents generated in the two subcells are identical. Taking the photocurrent of the top subcell PCDTBT:PC ${ }_{70} \mathrm{BM}$ into consideration, the resulting contour plot 
a

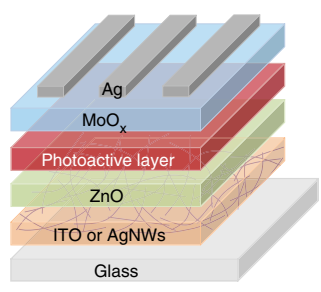

b

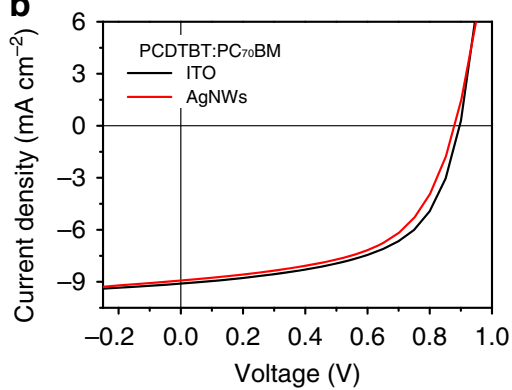

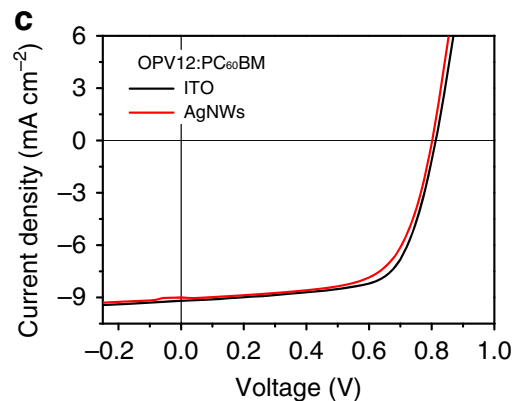

Figure 3 | Device structure and photovoltaic performance of single-junction cells. (a) Device architecture of inverted solar cells with $\mathrm{AgNW}$ bottom electrode. (b,c) Typical J-V curves of single-junction reference cells of PCDTBT:PC ${ }_{70} B M(\mathbf{b})$ and OPV12:PC 60 BM (c) deposited on ITO and AgNWs-coated glass substrates.
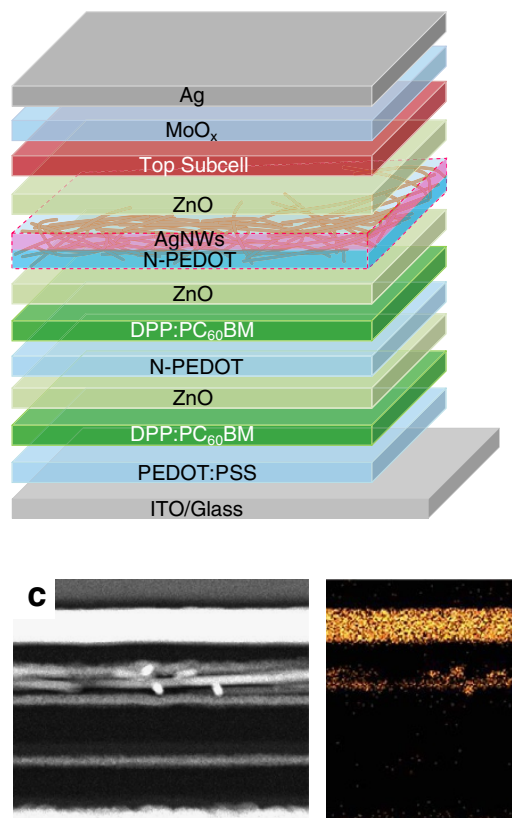

STIEM
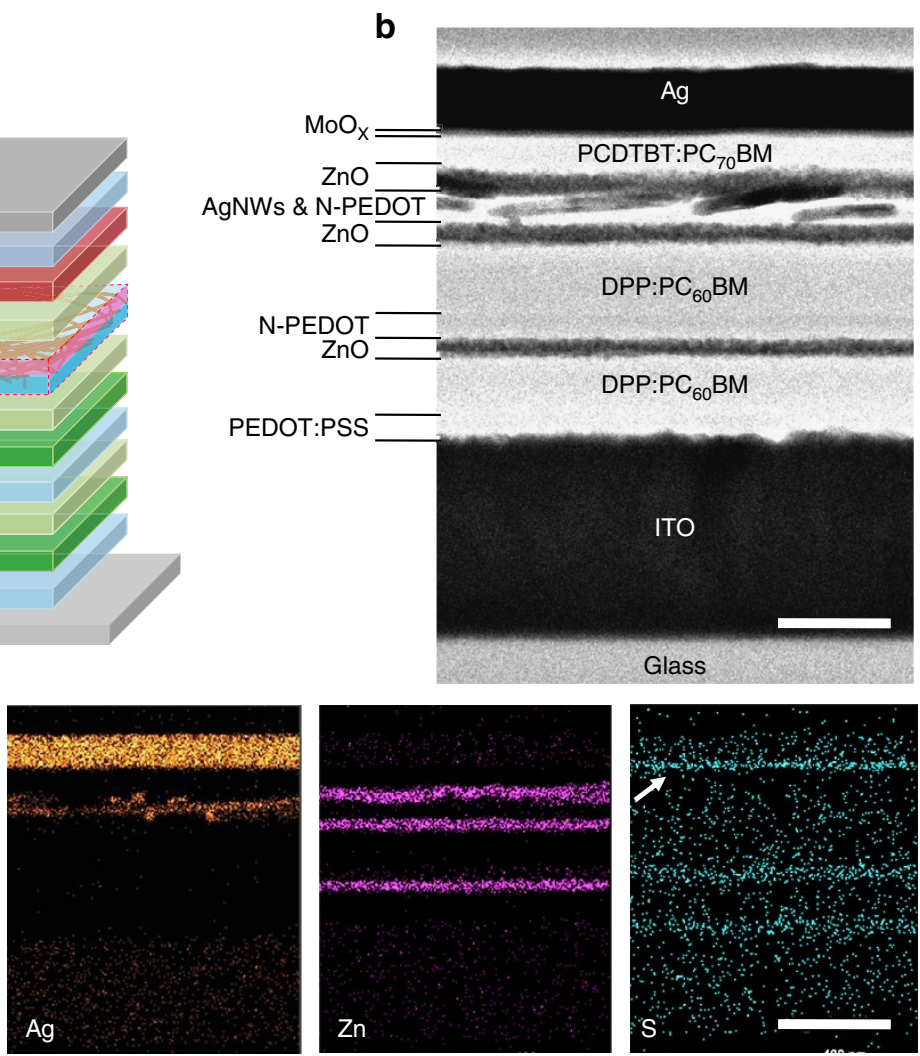

Figure 4 | Microstructure of the triple-junction solar cell. (a) Device architecture of the SP triple-junction solar cell. (b) A cross-sectional TEM image of the as-prepared triple-junction solar cell. The scale bar, $200 \mathrm{~nm}$. (c) STEM image of the cross-section and EDS elemental (Ag, Zn, S) maps. Note that the strongest top band (indicated by arrow) in the sulphur map belongs to molybdenum because of overlapping of S-K $\mathrm{K}^{\alpha}(2.307 \mathrm{keV})$ and Mo- $\mathrm{L}^{\alpha}$

$(2.293 \mathrm{keV})$ lines. The scale bar, $400 \mathrm{~nm}$.

of the current density distribution of the entire triple-junction solar cells as a function of the thicknesses of two DPP:PC ${ }_{60} \mathrm{BM}$ layers is depicted in Fig. 5b. One can see that maximum photocurrents of $\sim 10 \mathrm{~mA} \mathrm{~cm}^{-2}$ are achievable for our DPP-DPP/PCDTBT triple-junction devices when the thicknesses of the bottom and top DPP:PC ${ }_{60} \mathrm{BM}$ subcells are in the range of $30-60 \mathrm{~nm}$ and $35-80 \mathrm{~nm}$, respectively. Similar simulation results for the triple-junction DPP-DPP/OPV12 devices are presented in Supplementary Fig. 4.

Experimentally, to evaluate the photovoltaic performances of the subcells, we designed a three-terminal layout to prepare our SP triple-junction solar cells, which allows us to detect the $J-V$ characteristics of both the bottom series-tandem subcell and the top subcell within their connected state (Supplementary Fig. 3). Figure $5 c$,d show the typical $J-V$ curves of the constructed triple- junction solar cells, DPP-DPP/PCDTBT and DPP-DPP/OPV12, along with the constituent subcells, respectively. The key photovoltaic parameters are listed in Table 2. It can be seen that the two triple-junction cells achieved $J_{\mathrm{SC}}$ of $9.67 \mathrm{~mA} \mathrm{~cm}^{-2}$ (DPP-DPP/PCDTBT) and $9.55 \mathrm{~mA} \mathrm{~cm}^{-2}$ (DPP-DPP/OPV12) which is in good agreement with the optical simulations. Noticeably, from Table 2 we can see that the measured photocurrents of the triple-junction cells are more or less identical to the sum $J_{S C}$ values extracted from the respective bottom DPP-DPP subcells and top PCDTBT or OPV12 subcells. The $J_{\mathrm{SC}}$ values of the top subcells were verified with EQE measurement (Supplementary Fig. 5) and the values calculated by integrating the EQE curve with standard AM1.5 G spectrum show a good agreement with the measured $J_{S C}$ values. Moreover, as depicted in Fig. $5 c$,d, if we mathematically add the $J-V$ curves 
a
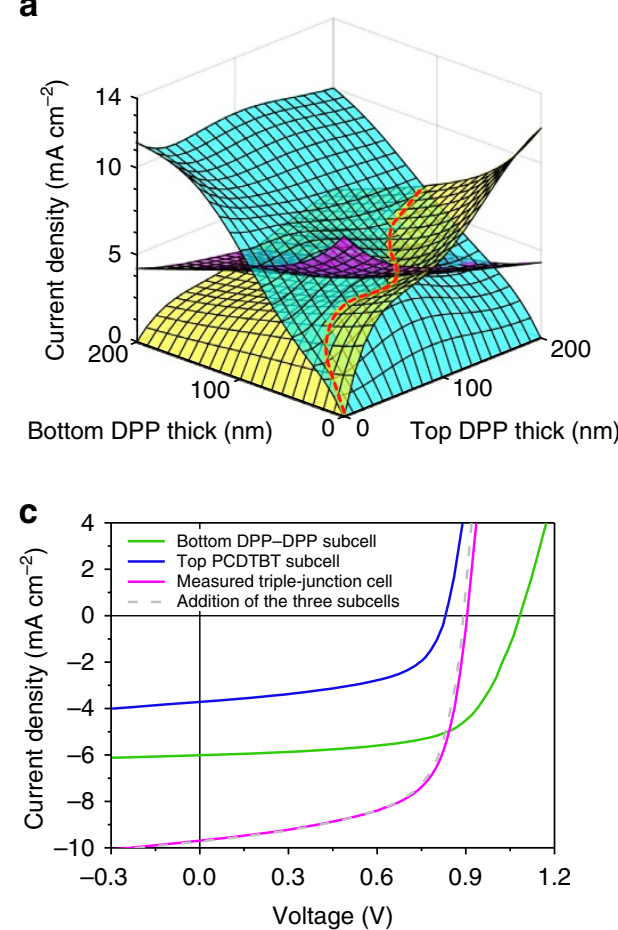

b
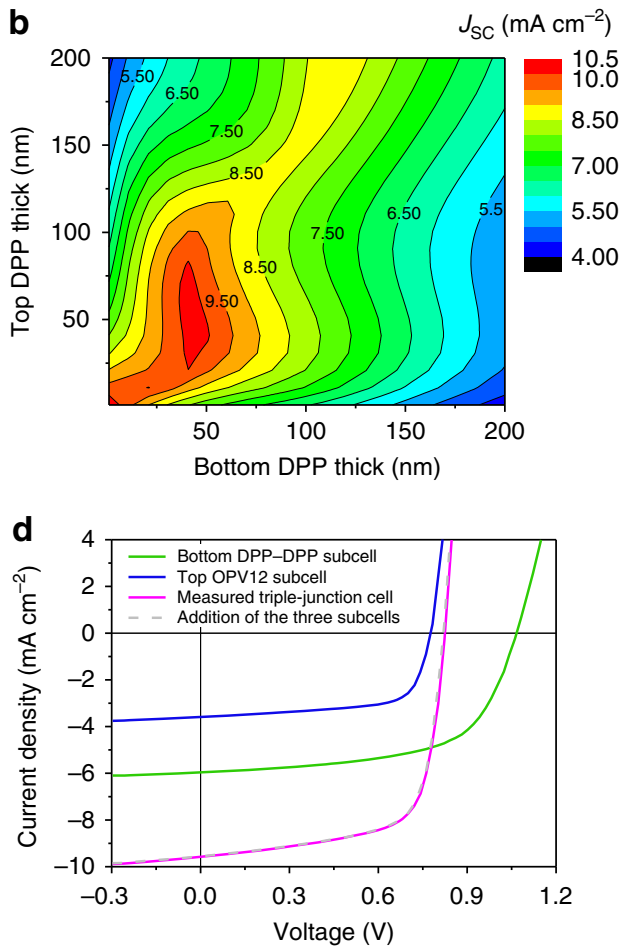

Figure 5 | Optical simulations and photovoltaic characteristics of the SP triple-junction solar cells. (a) Simulated current density distribution of the three subcells as a function of the thicknesses of bottom two DPP:PC ${ }_{60} B M$ layers. (b) Contour plot of current density distribution of the entire triplejunction devices (DPP-DPP/PCDTBT) as a function of the thicknesses of bottom DPP:PC ${ }_{60} B$ M layers. Note that in these two simulations the top PCDTBT:PC 70 BM layer thickness is fixed to $80 \mathrm{~nm}$, corresponding to the optimized thickness in their single-junction state. (c,d) $J-V$ characteristics of the investigated triple-junction cells and the constituent bottom series-tandem subcells and top subcell, (c) DPP-DPP/PCDTBT, (d) DPP-DPP/OPV12. The light grey dashed lines indicate the numerical addition of the bottom series-tandem subcells and the top subcell.

\begin{tabular}{|c|c|c|c|c|c|c|}
\hline $\begin{array}{l}\text { Material } \\
\text { combination }\end{array}$ & Devices & $\begin{array}{l}V_{\text {oc }} \\
\text { (V) }\end{array}$ & $\begin{array}{c}\mathbf{F F} \\
(\%)\end{array}$ & $\begin{array}{c}J_{S C} \\
\left(\mathrm{~mA} \mathrm{~cm}^{-2}\right)\end{array}$ & $\begin{array}{l}\text { PCE } \\
(\%)\end{array}$ & $\begin{array}{c}R_{\mathrm{S}} \\
\left(\Omega \mathrm{cm}^{2}\right)\end{array}$ \\
\hline \multirow{4}{*}{$\begin{array}{l}\text { DPP-DPP/ } \\
\text { PCDTBT }\end{array}$} & Bottom & 1.08 & 64.2 & 6.00 & 4.16 & 8.03 \\
\hline & $\begin{array}{l}\text { DPP-DPP } \\
\text { subcell }\end{array}$ & & & & & \\
\hline & $\begin{array}{l}\text { Top } \\
\text { PCDTBT } \\
\text { subcell }\end{array}$ & 0.82 & 54.9 & 3.70 & 1.66 & 1.15 \\
\hline & $\begin{array}{l}\text { Triple } \\
\text { connection }\end{array}$ & 0.89 & 63.1 & 9.67 & 5.43 & 1.04 \\
\hline \multirow{4}{*}{$\begin{array}{l}\text { DPP-DPP/ } \\
\text { OPV12 }\end{array}$} & Bottom & 1.07 & 60.7 & 5.95 & 3.86 & 7.14 \\
\hline & $\begin{array}{l}\text { DPP-DPP } \\
\text { subcell }\end{array}$ & & & & & \\
\hline & $\begin{array}{l}\text { Top OPV12 } \\
\text { subcell }\end{array}$ & 0.77 & 67.8 & 3.58 & 1.87 & 0.82 \\
\hline & $\begin{array}{l}\text { Triple } \\
\text { connection }\end{array}$ & 0.82 & 68.4 & 9.55 & 5.35 & 0.71 \\
\hline
\end{tabular}

DPP, pDPP5T-2; PCE, power conversion efficiency.

$R_{\mathrm{S}}$ of the cells were calculated at $\sim 2 \mathrm{~V}$ of the $J-V$ curves measured in dark.

of the DPP-DPP subcells with the top PCDTBT or OPV12 subcell at each voltage bias $\left(V_{\text {bias }}\right)$, a perfect fitting of the constructed $J-V$ curve with the experimentally measured $J-V$ curve of the triple-junction device is observed, which is consistent with Kirchhoff's law. These observations provide sufficient evidence that there are no resistive losses for the intermediate AgNW electrode in terms of collecting charge carriers.

For both triple-junction solar cells, the bottom seriesconnected DPP-DPP subcells showed $V_{\mathrm{OC}}$ values of
$1.07-1.08 \mathrm{~V}$, indicating that the solution-processing of the upper layers imposes no negative effect on the established bottom subcells. Compared with the reference DPP-DPP tandem cell, the slightly reduced $V_{\mathrm{OC}}$ of $0.02-0.03 \mathrm{~V}$ can be attributed to shadow effect $^{36}$, because a mask with an aperture smaller than either electrode was adopted to define the active area during the $J-V$ measurement. While the reduced light intensity filtered by the front DPP-DPP subcells further slightly decreased the $V_{\mathrm{OC}}$ of the back PCDTBT:PC ${ }_{70} \mathrm{BM}$ or OPV12:PC ${ }_{60} \mathrm{BM}$ subcells by a value of $0.03-0.05 \mathrm{~V}$.

For solar cells with ideal diode characteristics, the $V_{\mathrm{OC}}$ of the parallel-connected tandem cells would be strictly restricted by the subcell, which delivers low $V_{\text {OC. }}$. In real parallel-connected solar cells, however, the $V_{\mathrm{OC}}$ of the tandem cells can be close either to the subcell with high $V_{\mathrm{OC}}$ or to the subcell with low $V_{\mathrm{OC}}$ depending on the series resistance of the subcells ${ }^{37}$. In our parallel-connected constituent subcells, the two top subcells showed series resistance of $\sim 1 \Omega \mathrm{cm}^{2}$ which is almost eight times lower than those of bottom DPP-DPP subcells (Table 2). This is due to the fact that the charge injections in the top subcells are higher than in the bottom subcells at $V_{\text {bias }}>V_{\text {OC. }}$. Consequently, the top subcells showed steeper slopes at $V_{\text {bias }}>V_{\text {OC }}$ compared with the bottom subcells. Taking Kirchhoff's law into consideration, these circumstances lead to the $V_{\text {OC }}$ values of our triple-junction cells close to the top subcells which exhibited lower $V_{\text {OC. }}$.

Moreover, it should be noted that although our triple-junction cells have achieved PCEs of 5.35 and $5.43 \%$, which are higher than either one of the single-junction reference devices, those values are still $\sim 0.4 \%$ lower than the sum PCEs of the incorporated subcells. These PCE losses are mainly attributed to the relatively low $V_{\mathrm{OC}}$ of triple-junction that is close to the top 
subcells, and this suppression can be readily eliminated by employing high-performance top subcells with $V_{\mathrm{OC}}$ matched to the bottom series-connected subcells. Nevertheless, these results in combination with the high FFs of up to $68 \%$ eventually suggest that the engineered intermediate layers have efficiently coupled the three cells into triple-junction with an integrated SP interconnection.

Hybrid triple-junction solar cells. Organometal halide perovskites have emerged as promising materials that enable fabrication of highly efficient solar cells by solution deposition ${ }^{38-40}$. Although efficiencies exceeding $15 \%$ have been frequently reported, it is widely acknowledged that the moderate bandgap of $1.55 \mathrm{eV}$ offers enormous potential to further enhance the device efficiency by using multi-junction configuration ${ }^{39,40}$. Given that the perovskite single cell (mixed halide $\mathrm{CH}_{3} \mathrm{NH}_{3} \mathrm{PbI}_{3-x} \mathrm{Cl}_{x}$ ) provides a high $V_{\mathrm{OC}}$ of $\sim 1 \mathrm{~V}$, which is comparable to our series-connected DPP-DPP cells, it is straightforward to fabricate a PS connected triple-junction device by placing a DPP-DPP cell behind a semitransparent perovskite cell, and thereby adding up the total current density for the hybrid triple-junction device.

Figure 6a shows the calculated $J_{\mathrm{SC}}$ distribution of the three subcells of the hybrid triple-junction device as a function of the thicknesses of the back two DPP cells. We used an internal quantum efficiency of $100 \%$ for our simulation ${ }^{41}$. The front 200-nm-thick perovskite cell exhibits a $J_{\mathrm{SC}}$ of $\sim 16 \mathrm{~mA} \mathrm{~cm}^{-2}$, which is slightly affected by the interference of the device. The EQE measurement of a prepared semitransparent perovskite cell (Supplementary Fig. 6) gives a current density of $15.98 \mathrm{~mA} \mathrm{~cm}^{-2}$ which is in good agreement with the simulation values (Supplementary Methods for fabrication details). A current density of up to $3 \mathrm{~mA} \mathrm{~cm}^{-2}$ is calculated for the seriesconnected DPP-DPP tandem cell, as a benefit of the average $53.4 \%$ transmittance $(650$ and $850 \mathrm{~nm})$ of the semitransparent perovksite cell (Supplementary Fig. 7). Figure 6b shows the measured $J-V$ curves of the experimentally constructed hybrid triple-junction solar cell and the corresponding subcells. The semitransparent perovskite device shows a $J_{\mathrm{SC}}=16.28 \mathrm{~mA} \mathrm{~cm}^{-2}$, $V_{\mathrm{OC}}=0.94 \mathrm{~V}$ and $\mathrm{FF}=65.6 \%$, yielding a $\mathrm{PCE}$ of $10.04 \%$. The hybrid triple-junction device perovskite/DPP-DPP exhibits a high current density of $18.51 \mathrm{~mA} \mathrm{~cm}^{-2}$ with about $2 \mathrm{~mA} \mathrm{~cm}^{-2}$ contributed from the back DPP-DPP subcells. Together with the high $\mathrm{FF}$ of $64.5 \%$ and $V_{\mathrm{OC}}$ of $0.95 \mathrm{~V}$, the hybrid triple-junction device shows a PCE value of $11.34 \%$, corresponding to a PCE enhancement by $12.5 \%$.
To illustrate the benefit of the hybrid triple-junction device, we further theoretically compared the current generation between the single opaque perovskite cells and the hybrid triple-junction devices using the same material combinations. As presented in Fig. $6 c$, the $J_{\mathrm{SC}}$ value of the triple-junction device reaches to the $J_{\mathrm{SC}}$ value of the opaque single-junction perovskite cell, for perovskite cells with a layer thickness of $>300 \mathrm{~nm}$. It should be noted that the absorption of the DPP polymer donor shows a red-shift of only $\sim 50 \mathrm{~nm}$ compared with the perovskite and, therefore, we expect a significant enhancement when deeper NIR sensitizers are used as back series-connected tandem cells.

\section{Discussion}

We have experimentally demonstrated in this work, for the first time, solution-processed organic and hybrid triple-junction solar cells with integrated series- and parallel-interconnection. The optical simulations reveal that the as-proposed SP triple-junction organic solar cells hold the potential to achieve high efficiencies close to those of the fully series-connected counterparts, but allowing a much wider choice of material combinations. Through a rational interface layer design, triple-junction devices with all solution-processed intermediate layers achieved PCEs of $\sim 5.4 \%$ with FFs of up to $68 \%$. Currently, the efficiency of our SP triplejunction devices is mainly limited by the mismatch of the $V_{\mathrm{OC}}$ of the top subcell with the $V_{\mathrm{OC}}$ of the bottom series-connected tandem subcells. Based on the convenient solution-processing along with the impressive high FFs, we expect that significant enhancement in efficiency can be achieved by exploiting high-performance wide bandgap materials with matched $V_{\mathrm{OC}}$ in the back subcell. Alternatively, our results predict a significantly growing interest in ultra-low bandgap semiconductors allowing for more efficient light-harvesting for these SP triple-junction solar cells.

The general applicability of the proposed triple-junction configurations has also been verified in organic-inorganic hybrid triple-junction devices. By combining a semitransparent perovskite cell with series-connected DPP-DPP cells in parallel, the fabricated hybrid triple-junction devices showed an efficiency improvement by $12.5 \%$ compared with the corresponding reference cells. Further, we believe that the novel, but generic, concept demonstrated in this work potentially provides a promising avenue to approach or exceed the Shockley-Queisser limit of many of the currently available high-performance semiconductors such as crystalline silicon, $\mathrm{CdTe}$ and perovskite solar cells ${ }^{42-44}$. a

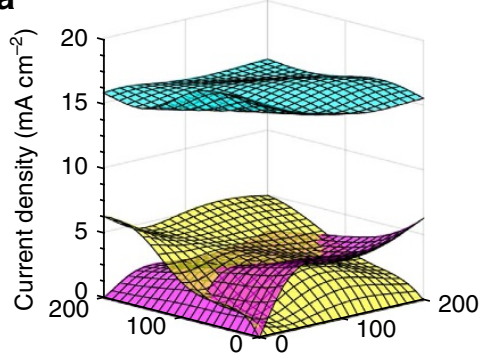

b

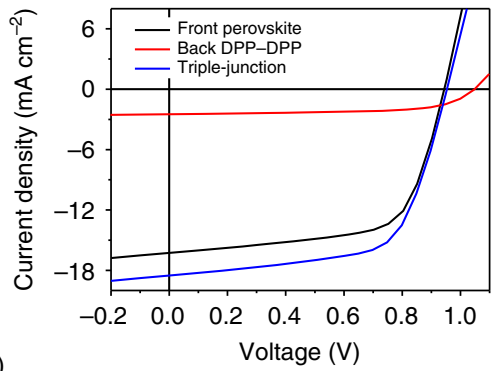

Voltage (V)
C

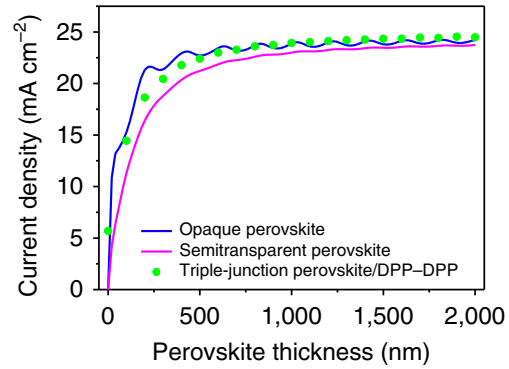

Front DPP thick $(\mathrm{nm}) \quad$ Back DPP thick $(\mathrm{nm})$

Figure 6 | Simulation and experimental demonstration of hybrid triple-junction solar cells with a parallel/series interconnection. (a) Calculated $J_{\mathrm{SC}}$ distribution of the three subcells as a function of the back two DPP:PC ${ }_{60} B M$ film thicknesses. The thickness of the front perovskite layer is fixed to 200 nm which corresponds to the thickness of the optimized reference cells. (b) Measured $J-V$ curves of the two constituent subcells and the triple-connected device. (c) Calculated $J_{S C}$ values of the semitransparent, opaque perovskite cells and the proposed triple-junction devices (perovskite/DPP-DPP) as a function of layer thickness of the perovskite. 


\section{Methods}

Materials. ITO-coated glass substrates $(2.5 \times 2.5) \mathrm{cm}^{2}$ with a sheet resistance of $15 \Omega \mathrm{sq}^{-1}$ were purchased from Weidner Glas and patterned with laser before use. Light absorbers DPP, OPV12 and PCDTBT were purchased from BASF, Polyera and 1-Materials, respectively. PEDOT:PSS (Clevios, P VP AI 4083) and N-PEDOT (NT5-3417286/2) were obtained from Heraeus and Agfa, respectively. ZnO nanoparticles dispersed in isopropanol (Product N-10) and AgNW dispersion (ClearOhm Ink) were supplied by Nanograde AG and Cambrios Technologies Corporation, respectively. $\mathrm{PC}_{60} \mathrm{BM}(99.5 \%)$ and $\mathrm{PC}_{70} \mathrm{BM}(99 \%)$ were purchased from Solenne BV. All the materials were used as received without further purification.

Fabrication of triple-junction devices. For our SP triple-junction organic solar cells, with the exception of bottom ITO-coated glass substrate and top evaporated $\mathrm{MoO}_{X} / \mathrm{Ag}$ electrode, all the layers were sequentially deposited using a doctor blade in ambient atmosphere. Prior to device fabrication, the laser-patterned ITO substrates were cleaned by ultra-sonication in acetone and isopropanol for $10 \mathrm{~min}$ each.

On the cleaned substrates, PEDOT:PSS (Clevious P VP Al 4083, 1:3 vol.\% diluted in isopropanol) was firstly bladed and annealed at $140^{\circ} \mathrm{C}$ for $5 \mathrm{~min}$ to obtain a layer thickness of $\sim 40 \mathrm{~nm}$. On top of the dried PEDOT:PSS, the first photoactive layer consisting of DPP and $\mathrm{PC}_{60} \mathrm{BM}(1: 2 \mathrm{wt} . \%$ dissolved in a mixed solvent of chloroform and o-dichlorobenzene $(9: 1 \mathrm{vol} . \%)$ ) was deposited at $45^{\circ} \mathrm{C}$ to obtain a thickness of $\sim 50 \mathrm{~nm}$. The first intermediate layers, $\mathrm{ZnO}$ and N-PEDOT:PSS, were sequentially bladed at $50^{\circ} \mathrm{C}$ and annealed at $80^{\circ} \mathrm{C}$ for $5 \mathrm{~min}$ in air and the obtained layer thickness for both layers is $\sim 35 \mathrm{~nm}$. The second active layer DPP:PC ${ }_{60} \mathrm{BM}$ with thickness of $\sim 80 \mathrm{~nm}$ was then coated on top of $\mathrm{N}$-PEDOT at $55^{\circ} \mathrm{C}$. Afterwards, $\mathrm{ZnO}$ and N-PEDOT were again deposited onto the second DPP:PC ${ }_{60} \mathrm{BM}$ layer using the same coating parameters as for the first deposition. To deposit the intermediate electrode, $\sim 80-\mathrm{nm}$-thick AgNWs was bladed onto N-PEDOT at $45^{\circ} \mathrm{C}$ and the resulting NW film showed a sheet resistance of $\sim 8 \Omega \mathrm{sq}^{-1}$. Successively, an electron extraction layer of $\mathrm{ZnO}$ was deposited on top of AgNWs using the same parameters, followed by blading the third active blend of PCDTBT:PC ${ }_{70} \mathrm{BM}$ at $60^{\circ} \mathrm{C}$. After all the solution-processed layers were completed, Q-tips dipped with toluene were used to clean the edges of the substrate to expose the bottom ITO and middle AgNW contacts. Finally, to complete the device fabrication, a 15-nm-thick $\mathrm{MoO}_{X}$ and $100-\mathrm{nm}$-thick $\mathrm{Ag}$ were thermally evaporated on top of PCDTBT:PC ${ }_{70} \mathrm{BM}$ through a shadow mask with an opening of $10.4 \mathrm{~mm}^{2}$.

Triple-junction solar cells DPP-DPP/OPV12 were prepared with the same processing procedure as device DPP-DPP/PCDTBT. To achieve a reliable contact between the middle AgNW electrode and probes of the measurement set-ups ( $J-V$ and EQE measurements), silver paste or evaporated silver was applied to the exposed AgNWs (Supplementary Fig. 3). Semitransparent DPP-DPP reference tandem cells with top AgNW electrode and the single-junction reference devices (PCDTBT:PC 70 BM and OPV12: $\mathrm{PC}_{60} \mathrm{BM}$ ) with bottom AgNW electrode were fabricated using the same procedure as these subcells in the SP triple-junction cells.

The semitransparent perovskite (mixed halide $\mathrm{CH}_{3} \mathrm{NH}_{3} \mathrm{PbI}_{3-x} \mathrm{Cl}_{x}$ ) solar cells with a device structure of ITO/PEDOT:PSS/Perovskite/PC ${ }_{60} \mathrm{BM} / \mathrm{ZnO} / \mathrm{AgNWs}$ (Supplementary Fig. 5a) was fabricated using a procedure as described in the Supplementary Methods ${ }^{45}$. The hybrid triple-junction solar cell was assembled by stacking a series-connected opaque DPP-DPP as back subcell with a semitransparent perovskite device as front subcell. The parallel-connection between the semitransparent perovskite and series-connected DPP-DPP subcells was realized by external coupling using $\mathrm{Ag}$ paste.

Characterization. Transmittance spectra of the intermediate layers and semitransparent devices were measured using a UV-vis-NIR spectrometer (Lambda 950, from Perkin Elmer). $J-V$ curves of all the devices were recorded using a source measurement unit from BoTest. Illumination was provided by a solar simulator (Oriel Sol $1 \mathrm{~A}$ from Newport) with AM1.5G spectrum and light intensity of $100 \mathrm{~mW} \mathrm{~cm}^{-2}$, which was calibrated by a certified silicon solar cell. To guarantee the incident light to be able to illuminate on all the three electrodes with an overlapped active area, during the $J-V$ measurement a mask with an aperture of $4.5 \mathrm{~mm}^{2}$ was used to define the cell area. The EQE spectra were recorded with an EQE measurement system (QE-R) from Enli Technology (Taiwan). The light intensity at each wavelength was calibrated with a standard single-crystal Si solar cell. Liftout sample for TEM was prepared with FEI Helios Nanolab 660 DualBeam FIB, from the area-of-interest containing all layers of the solar cell. A lamella containing a cross-section of the solar cell was then attached to a TEM half grid for final thinning. The final thickness of the liftout sample was kept $<100 \mathrm{~nm}$, to enable high quality conventional transmission electron microscopy (CTEM) imaging at an acceleration voltage of $200 \mathrm{kV}$. TEM was performed on the FEI TITAN $^{3}$ Themis 60-300 double aberration-corrected microscope at the Center for Nanoanalysis and Electron Microscopy (CENEM), the University of Erlangen, equipped with the super-X energy dispersive spectrometer.

\section{References}

1. Shockley, W. \& Queisser, H. J. Detailed balance limit of efficiency of p-n junction solar cells. J. Appl. Phys. 32, 510-519 (1961).
2. Green, M. A., Emery, K., Hishikawa, Y., Warta, W. \& Dunlop, E. D. Solar cell efficiency tables (Version 45). Prog. Photovoltaics 23, 1-9 (2015).

3. Trupke, T., Green, M. A. \& Wurfel, P. Improving solar cell efficiencies by up-conversion of sub-band-gap light. J. Appl. Phys. 92, 4117-4122 (2002).

4. Devos, A. Detailed balance limit of the efficiency of tandem solar-cells. J. Phys. D. Appl. Phys. 13, 839-846 (1980).

5. Trupke, T. \& Wurfel, P. Improved spectral robustness of triple tandem solar cells by combined series/parallel interconnection. J. Appl. Phys. 96, 2347-2351 (2004).

6. You, J. B. et al. A polymer tandem solar cell with $10.6 \%$ power conversion efficiency. Nat. Commun. 4, 1446 (2013).

7. Semonin, O. E. et al. Peak external photocurrent quantum efficiency exceeding $100 \%$ via MEG in a quantum dot solar cell. Science 334, 1530-1533 (2011).

8. Luque, A., Marti, A. \& Nozik, A. J. Solar cells based on quantum dots: Multiple exciton generation and intermediate bands. MRS Bull. 32, 236-241 (2007).

9. Hirst, L. C. \& Ekins-Daukes, N. J. Fundamental losses in solar cells. Prog. Photovoltaics 19, 286-293 (2011).

10. Hendriks, K. H., Li, W. W., Wienk, M. M. \& Janssen, R. A. J. Small-bandgap semiconducting polymers with high near-infrared photoresponse. J. Am. Chem. Soc. 136, 12130-12136 (2014).

11. Ashraf, R. S. et al. Chalcogenophene comonomer comparison in small band gap diketopyrrolopyrrole-based conjugated polymers for high-performing fieldeffect transistors and organic solar cells. J. Am. Chem. Soc. 137, 1314-1321 (2015).

12. Tang, J. et al. Quantum junction solar cells. Nano Lett. 12, 4889-4894 (2012).

13. Zhao, N. et al. Colloidal PbS quantum dot solar cells with high fill factor. ACS Nano 4, 3743-3752 (2010).

14. Dennler, G. et al. Design rules for donors in bulk-heterojunction tandem solar cells-towards $15 \%$ energy-conversion efficiency. Adv. Mater. 20, 579-583 (2008).

15. Scharber, M. C. et al. Design rules for donors in bulk-heterojunction solar cells - Towards 10\% energy-conversion efficiency. Adv. Mater. 18, 789-794 (2006).

16. Guo, F. et al. Solution-processed parallel tandem polymer solar cells using silver nanowires as intermediate electrode. ACS Nano 8, 12632-12640 (2014).

17. Guo, F. et al. Fully solution-processing route toward highly transparent polymer solar cells. ACS Appl. Mater. Interface 6, 18251-18257 (2014).

18. Adebanjo, O. et al. Triple junction polymer solar cells. Energy Environ. Sci. 6, 3150-3170 (2013).

19. Chen, C. C. et al. An efficient triple-junction polymer solar cell having a power conversion efficiency exceeding 11\%. Adv. Mater. 26, 5670-5677 (2014).

20. Dou, L. T. et al. Tandem polymer solar cells featuring a spectrally matched lowbandgap polymer. Nat. Photonics 6, 180-185 (2012).

21. Gevaerts, V. S., Furlan, A., Wienk, M. M., Turbiez, M. \& Janssen, R. A. J. Solution processed polymer tandem solar cell using efficient small and wide bandgap polymer:fullerene blends. Adv. Mater. 24, 2130-2134 (2012).

22. Kim, J. Y. et al. Efficient tandem polymer solar cells fabricated by all-solution processing. Science 317, 222-225 (2007).

23. Li, N. et al. An efficient solution-processed intermediate layer for facilitating fabrication of organic multi-junction solar cells. Adv. Energy Mater. 3, 1597-1605 (2013).

24. Li, N. et al. Environmentally printing efficient organic tandem solar cells with high fill factors: a guideline towards $20 \%$ power conversion efficiency. Adv. Energy Mater. 4, 1400084 (2014).

25. Li, W. W., Furlan, A., Hendriks, K. H., Wienk, M. M. \& Janssen, R. A. J. Efficient tandem and triple-junction polymer solar cells. J. Am. Chem. Soc. 135, 5529-5532 (2013).

26. Lee, J. Y., Connor, S. T., Cui, Y. \& Peumans, P. Solution-processed metal nanowire mesh transparent electrodes. Nano Lett. 8, 689-692 (2008).

27. Leem, D. S. et al. Efficient organic solar cells with solution-processed silver nanowire electrodes. Adv. Mater. 23, 4371-4375 (2011).

28. Guo, F. et al. ITO-free and fully solution-processed semitransparent organic solar cells with high fill factors. Adv. Energy Mater. 3, 1062-1067 (2013).

29. Song, M. et al. Highly efficient and bendable organic solar cells with solutionprocessed silver nanowire electrodes. Adv. Funct. Mater. 23, 4177-4184 (2013).

30. Beiley, Z. M. et al. Semi-transparent polymer solar cells with excellent sub-bandgap transmission for third generation photovoltaics. Adv. Mater. 25, 7020-7026 (2013).

31. Sista, S., Hong, Z. R., Park, M. H., Xu, Z. \& Yang, Y. High-efficiency polymer tandem solar cells with three-terminal structure. Adv. Mater. 22, E77-E80 (2010).

32. Zuo, L. J. et al. Microcavity-enhanced light-trapping for highly efficient organic parallel tandem solar cells. Adv. Mater. 26, 6778-6784 (2014).

33. Li, N. et al. Towards $15 \%$ energy conversion efficiency: a systematic study of the solution-processed organic tandem solar cells based on commercially available materials. Energy Environ. Sci. 6, 3407-3413 (2013).

34. Pettersson, L. A. A., Roman, L. S. \& Inganas, O. Modeling photocurrent action spectra of photovoltaic devices based on organic thin films. J. Appl. Phys. 86, 487-496 (1999). 
35. Centurioni, E. Generalized matrix method for calculation of internal light energy flux in mixed coherent and incoherent multilayers. Appl. Opt. 44, $7532-7539$ (2005).

36. Silvestre, S. \& Chouder, A. Effects of shadowing on to photovoltaic module performance. Prog. Photovoltaics Res. Appl. 16, 141-149 (2008).

37. Hadipour, A., de Boer, B. \& Blom, P. W. M. Device operation of organic tandem solar cells. Org. Electron. 9, 617-624 (2008).

38. Kojima, A., Teshima, K., Shirai, Y. \& Miyasaka, T. Organometal halide perovskites as visible-light sensitizers for photovoltaic cells. J. Am. Chem. Soc. 131, 6050-6051 (2009).

39. Green, M. A., Ho-Baillie, A. \& Snaith, H. J. The emergence of perovskite solar cells. Nat. Photonics 8, 506-514 (2014).

40. Snaith, H. J. Perovskites: the emergence of a new era for low-cost, high-efficiency solar cells. J. Phys. Chem. Lett. 4, 3623-3630 (2013).

41. Sun, S. Y. et al. The origin of high efficiency in low-temperature solutionprocessable bilayer organometal halide hybrid solar cells. Energy Environ. Sci. 7, 399-407 (2014).

42. Kim, T. et al. Triple-junction hybrid tandem solar cells with amorphous silicon and polymer-fullerene blends. Sci. Rep. 4, 7154 (2014).

43. Beiley, Z. M. \& McGehee, M. D. Modeling low cost hybrid tandem photovoltaics with the potential for efficiencies exceeding 20\%. Energy Environ. Sci. 5, 9173-9179 (2012).

44. Kim, J. et al. $10.5 \%$ efficient polymer and amorphous silicon hybrid tandem photovoltaic cell. Nat. Commun. 6, 6391 (2015).

45. Guo, F. et al. High-performance semitransparent perovskite solar cells with solution-processed silver nanowires as top electrodes. Nanoscale 7, 1642-1649 (2015).

\section{Acknowledgements}

The work was supported by the Cluster of Excellence 'Engineering of Advanced Materials' (EAM) and the SFB 953 at the University of Erlangen-Nuremberg. We would like to thank Cambrios Technology Corporation, Dr Mathieu Turbiez from BASF and Dr Norman Lüchinger from Nanograde for the supply of AgNWs, DPP and ZnO dispersion, respectively. V.R.R. and V.V.R. acknowledge financial support from the
Ministry of Education, Science and Technological Development of the Republic of Serbia (Grants No. 172054 and No. III45019, respectively.) and from the DFG research training group GRK 1896 at the Erlangen University. F.G. and N.L. would like to acknowledge the funding from the China Scholarship Council and the Joint Project Helmholtz-Institute Erlangen Nürnberg (HI-ERN) under project number DBF01253, respectively. K.F. and C.J.B. gratefully acknowledge the financial support through the 'Aufbruch Bayern' initiative of the state of Bavaria.

\section{Author contributions}

F.G. and C.J.B. conceived the device concept. F.G., N.L. and N.G. fabricated and characterized the organic solar cells. C.O.R.Q., C.B. and Y.H. prepared the semitransparent perovskite cells. F.W.F. carried out the semi-empirical modelling. F.G. and K.F. performed the optical simulations. V.V.R., V.R.R. and E.S. prepared the FIB sample and performed the TEM imaging. C.J.B., F.G. and N.L. contributed to project planning and manuscript preparation. All the authors commented on the manuscript.

\section{Additional information}

Supplementary Information accompanies this paper at http://www.nature.com/ naturecommunications

Competing financial interests: The authors declare no competing financial interests.

Reprints and permission information is available online at http://npg.nature.com/ reprintsandpermissions/

How to cite this article: Guo, F. et al. A generic concept to overcome bandgap limitations for designing highly efficient multi-junction photovoltaic cells. Nat. Commun 6:7730 doi: 10.1038/ncomms8730 (2015).

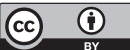

This work is licensed under a Creative Commons Attribution 4.0 International License. The images or other third party material in this article are included in the article's Creative Commons license, unless indicated otherwise in the credit line; if the material is not included under the Creative Commons license, users will need to obtain permission from the license holder to reproduce the material. To view a copy of this license, visit http://creativecommons.org/licenses/by/4.0/ 